\title{
MICROSCOPY
}

\section{Keep still! Immobilizing animals with hydrogels}

\author{
Burnett, K., Edsinger, E., and Albrecht D.R. Commun. Biol. 1, 73 (2018)
}

To image a living animal under a microscope for an extended period of time, it needs to be kept still. Dirk Albrecht works with the nematode Caenorhabditis elegans in his quantitative neurotechnology lab at Worcester Polytechnic Institute in Massachusetts, and he found that the existing approaches to immobilizing the worm under his microscopes weren't quite doing the trick. The animals are quite strong for their size, he notes, and the agarose gels that might hold a zebrafish embryo more or less in place couldn't contain C. elegans well enough to meet his long-term imaging needs. He needed an alternative.

From prior bioengineering days, he thought about hydrogels. They aren't a new medium and their properties are well documented: they have refractive index close to water, as the name suggests; their covalent cross-links are strong but easy to fine-tune; and they're biologically inert. The material is cheap too-about a penny per microliter. Researchers have used hydrogels for decades to embed mammalian cells, Albrecht says. Why not try a whole animal?

He narrowed in on one in particular, polyethylene-glycol (PEG) hydrogel. Some hydrogels need to be heated to get them to gel-this can negatively impact animals that are sensitive to heat, such as C. elegans. Others are ionic, but changing salt concentrations to set the hydrogel can be a problem for animals with narrow salt tolerances. But with the addition of a photoinitator, PEG hydrogel can be activated with just a little light and it will set at any temperature or salinity, he explains.

Albrecht and his lab tried out different formulations until they came up with one that they felt worked best to immobilize C. elegans. The basic steps are pretty simple. From the lab's latest publication in Communications Biology, just pipette a few microliters of PEG hydrogel solution onto a slide with spacers added, gently place a worm in the solution, cover, and expose to a few seconds of light.

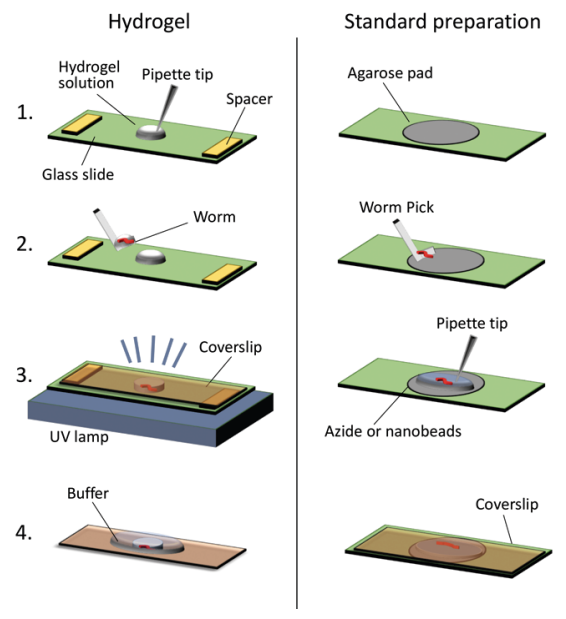

Hydrogel vs. agarose mounting. Image adapted from Burnett, K., Edsinger, E., and Albrecht D.R. (2018), Springer Nature.

Et voila, a worm held almost entirely still for up to 24 hours. When all the images needed are collected, a quick prick with a pick or forceps will release the animal. In the results presented in the paper, $86 \%$ of the worms crawled away unfazed by their time encapsulated. The authors speculate that most of those that didn't were damaged by the forceps or by internal egg hatching.

The technique worked well under an upright light microscope as well as with a light sheet microscope, where the embedding medium needs to have a refractive index close to that of water.

It's also tunable for immobilizing much more than just C. elegans. "With a little bit of optimization, it's worked for just about everything we've done," Albrecht says. For the paper, the authors successfully encapsulated three-day old pygmy squid hatchlings in the hydrogel. The squid are larger than C. elegans and their soft bodies can be harmed if too tightly squeezed between coverslips, he says.

Albrecht found his squid collaborator, Eric Edsinger, during a meeting at the Marine Biological Laboratory in Woods Hole, Massachusetts. Others who've seen
Albrecht demonstrate the technique have wanted to try it out, and organisms from yeast to flies to zebrafish have all been encapsulated since, he says. His lab may have developed the technique to solve specific problems for their own research, he explains, "but that it is useful to everyone else is I think, awesome."

Jan Huisken, a researcher at the Morgridge Institute for Research in Wisconsin who was not involved with the current study, says he's eager to test out the hydrogel. "While this methods works very well only for a certain group of small and agile animals, it certainly enlarges our arsenal of embedding techniques. I especially like the fact that the gelation can be initiated quickly with UV light. This way the actual sample encapsulation can be triggered in the microscope at any given time once the animal has reached a certain location or adopted a certain shape or orientation," he commented via email.

Each animal is a little different, so it can take some tweaking to figure out the best formulation to use. What concentration of hydrogel is best? How thick does it need to be applied? Does the animal need any anesthetic to keep it extra still? To ease any confusion, Albrecht and his collaborators are starting to write out additional protocols to help interested but unfamiliar researchers determine the optimal starting point for their own organism and particular imaging needs.

Albrecht is taking advantage of hydrogels back in his own lab too. Because its porosity and thickness can be manipulated, he is looking in to what kinds of proteins or small molecules he can diffuse into the hydrogel to perturb physiological responses in C. elegans. He'd like to add something like an enzyme or a drug, observe its effect on the worm, and then wash it away to see what happens to the animal afterwards.

\section{Ellen P. Neff}

Published online: 24 August 2018 https://doi.org/10.1038/s41684-018-0146-0 